\title{
SIMULTANEOUS KERNELS OF MATRIX HADAMARD POWERS
}

\author{
ALEXANDER BELTON, DOMINIQUE GUILLOT, APOORVA KHARE, AND MIHAI PUTINAR
}

\begin{abstract}
In previous work [Adv. Math. 298:325-368, 2016], the structure of the simultaneous kernels of Hadamard powers of any positive semidefinite matrix was described. Key ingredients in the proof included a novel stratification of the cone of positive semidefinite matrices and a well-known theorem of Hershkowitz, Neumann, and Schneider, which classifies the Hermitian positive semidefinite matrices whose entries are 0 or 1 in modulus. In this paper, we show that each of these results extends to a larger class of matrices which we term 3-PMP (principal minor positive).
\end{abstract}

\section{INTRODUCTION}

Given a positive integer $N$ and a subset $I \subset \mathbb{C}$, let $\mathcal{P}_{N}(I)$ denote the collection of $N \times N$ Hermitian positive semidefinite matrices with all entries in $I$. Motivated by the study of entrywise transformations of a matrix which preserve positivity, the authors computed in [2] the simultaneous kernel $\mathcal{K}(A)$ of Hadamard powers of a matrix $A=\left(a_{i j}\right) \in \mathcal{P}_{N}(\mathbb{C})$ : that is,

$$
\mathcal{K}(A):=\bigcap_{n \geq 0} \operatorname{ker} A^{\circ n},
$$

where $A^{\circ n}:=\left(a_{i j}^{n}\right)$. Here, we use the convention that $A^{\circ 0}:=\mathbf{1}_{N}$, the rank-one $N \times N$ matrix with all entries equal to 1 . Note that when the entrywise powers are replaced by the conventional matrix powers and when $A$ is Hermitian, the kernels ker $A^{n}$ are all equal to $\operatorname{ker} A$ for $n \geq 1$. In contrast, the simultaneous kernels of Hadamard powers are highly structured.

Theorem 1.1 (see [2, Theorem 5.7]). Let $A \in \mathcal{P}_{N}(\mathbb{C}) \backslash\{0\}$ and let $c_{0}, \ldots, c_{N-1} \in(0, \infty)$. Then

$$
\mathcal{K}(A)=\operatorname{ker}\left(\sum_{j=0}^{N-1} c_{j} A^{\circ j}\right)=\bigcap_{n=0}^{N-1} \operatorname{ker} A^{\circ n}=\bigoplus_{j=1}^{m} \operatorname{ker} \mathbf{1}_{I_{j}}
$$

where $\pi^{\{1\}}(A):=\left\{I_{1}, \ldots, I_{m}\right\}$ is a partition of $\{1, \ldots, N\}$ whose construction is described in Section 因.

The matrix $\sum_{j=0}^{N-1} c_{j} A^{\circ j}$ is obtained by applying the polynomial $p(x)=\sum_{j=0}^{N-1} c_{j} x^{j}$ to the entries of $A$. The first equality thus provides a connection between the study of simultaneous kernels and the study of polynomials that preserve positivity when applied entrywise to matrices in $\mathcal{P}_{N}(\mathbb{C})$; see [2, 3] for more details.

An intriguing consequence of Theorem 1.1 is the rigidity of the simultaneous kernels: there are only finitely many possibilities for $\mathcal{K}(A)$. This is in stark contrast to the case where Hadamard powers are replaced by conventional powers, in which case any subspace of $\mathbb{C}^{N}$ can obviously arise as a simultaneous kernel.

Date: October 18th, 2017.

2010 Mathematics Subject Classification. 15B48 (primary); 15A21 (secondary).

Key words and phrases. Positive semidefinite matrix, Hadamard product, Schubert cell-type stratification, principal minor positive, principal submatrix rank property, simultaneous kernel. 
The proof of Theorem 1.1 in [2] was lengthy and involved, with two main ingredients.

(a) An observation of Hershkowitz, Neumann, and Schneider [7, which classifies Hermitian positive semidefinite matrices whose entries are 0 or 1 in modulus.

(b) A novel Schubert cell-type stratification for Hermitian positive semidefinite matrices 2, Theorem 5.1].

We show in the present note how these two ingredients, as well as Theorem 1.1. can be extended to a much broader class of Hermitian matrices. Namely, we show that these results hold for any matrix whose principal minors of size at most 3 are non-negative. Matrices satisfying the latter property will be termed 3-PMP (principal minor positive). Apart from proving Theorem 1.1] in greater generality, our new approach simplifies the original proof [2].

The article is structured as follows. In Section 2, we recall the main result of HershkowitzNeumann-Schneider [7] and extend it to 3-PMP matrices. A key component of their original proof is the principal submatrix rank property (PSRP). We therefore examine the relationship between the PSRP and the notion of principal minor positivity. In Section 3, we identify precisely how principal minor positivity constrains the signature of a Hermitian matrix. In Section 4, we recall and extend to all Hermitian matrices the Schubert cell-type stratification of the cone of positive semidefinite matrices that was developed in [2]. Section 5 concludes the paper by classifying the simultaneous kernels of Hadamard powers of all 3-PMP matrices.

\section{The Hershkowitz-Neumann-Schneider theorem And the Principal submatrix RANK PROPERTY}

We start by isolating the main class of matrices of interest in this paper.

Definition 2.1. Let $1 \leq k \leq N$. A Hermitian matrix $A \in \mathbb{C}^{N \times N}$ will be termed $k$-PMP if every principal $j \times j$ minor of $A$ is non-negative, for $j=1, \ldots, k$.

The notion of principal minor positivity interpolates between Hermitian matrices, which are 0-PMP by convention, and positive semidefinite matrices, which are $N$-PMP.

Remark 2.2 (Examples and special cases). Given $N \geq 1$, let $A:=\lambda \operatorname{Id}_{N}-\mathbf{1}_{N}$, where $\operatorname{Id}_{N}$ is the $N \times N$ identity matrix and $\mathbf{1}_{N}$ is the rank-one $N \times N$ matrix with all entries equal to 1 . It is readily seen that if $k \in\{1, \ldots, N\}$ and $\lambda \in[k-1, k)$, then the matrix $A$ is $(k-1)$-PMP but not $k$-PMP. Thus the successive inclusions in the PMP-filtration of all Hermitian matrices are strict. R. B. Bapat pointed us to work of Mohan, Parthasarathy, and Sridhar, who introduced the notion of P-matrices of exact order $N-k$; see [10]. These are a refinement of $k$-PMP matrices, whose principal minors of size no more than $k$ are positive, and those of size greater than $k$ are negative. The matrix $A:=\lambda \operatorname{Id}_{N}-\mathbf{1}_{N}$ is a $P$-matrix of exact order $N-k$ whenever $\lambda \in(k-1, k)$.

Our goal in this paper is to compute the simultaneous kernel for the entrywise powers of any given 3-PMP matrix; these comprise a much larger family of matrices than the cone $\mathcal{P}_{N}(\mathbb{C})$ that was considered in [2]. As noted in the Introduction, the following Theorem is an important first step.

Definition 2.3 (Hershkowitz-Neumann-Schneider [7, Definition 2.1]). A matrix $P \in \mathbb{C}^{N \times N}$ is called a unitary monomial matrix if $P=Q D$, where $Q$ is a permutation matrix and $D$ is a diagonal matrix all of whose diagonal entries are of modulus 1.

Theorem 2.4 (Hershkowitz-Neumann-Schneider [7, Theorem 2.2]). A matrix $A \in \mathbb{C}^{N \times N}$ is Hermitian positive semidefinite and all its entries have modulus 1 or 0 if and only if $A$ is 
similar, by means of a unitary monomial matrix, to a direct sum of matrices each of which is either a matrix with entries all equal to 1 or a zero matrix.

Positive semidefinite matrices with entries in $S^{1} \sqcup\{0\}$, as well as 3-PMP matrices, occur in many settings. For example, they naturally feature in Horn's study of the incidence matrices of infinitely divisible matrices and kernels [8, Theorem 1.13]. A simple application of Theorem 2.4 also shows that graphs with smallest adjacency eigenvalue -1 are disjoint unions of complete graphs [5]. Properties of matrix thresholding were also derived in [6] using related ideas.

Remark 2.5. It is worth mentioning that the unitary monomial matrices of Definition 2.3 were discovered by Banach in connection with a typical early functional-analysis question. More precisely, these matrices appear in the structure of the group $\mathcal{G}_{p}$ of linear isometries of $\mathbb{R}^{N}$ or $\mathbb{C}^{N}$ equipped with the $\ell^{p}$ norm, for $p \in[1, \infty]$. (Thanks to the Mazur-Ulam theorem, in the real case every isometry belongs to this group, up to a translation; the existence of the canonical conjugation shows this is false in the complex case.)

As is well known, the group $\mathcal{G}_{2}$ is the orthogonal or unitary group, respectively. If $p \neq 2$, then results of Banach and Lamperti [1, 9] imply that the group $\mathcal{G}_{p}$ does not depend on $p$, and consists of precisely the generalized permutation matrices, i.e., the products of permutation matrices with diagonal orthogonal or unitary matrices. Specifically, for $\mathbb{R}^{N}$ we obtain the hyperoctahedral group of $2^{N} N$ ! signed permutations, i.e., the Weyl group of type $B_{N}=C_{N}$. In the complex case, the group $\mathcal{G}_{p}$ is composed of all matrices which are products of permutations and diagonal unitary matrices, i.e., the unitary monomial matrices. (Elementary proofs of these special cases of the Banach-Lamperti theorem have been found.)

Returning to Theorem 2.4, the proof by Hershkowitz, Neumann, and Schneider is rather intriguing, relying on the following notion.

Definition 2.6. A matrix $M \in \mathbb{C}^{N \times N}$ is said to satisfy the principal submatrix rank property $(P S R P)$ if the following conditions hold.

(1) The column space determined by every set of rows of $M$ is equal to the column space of the principal submatrix lying in these rows.

(2) The row space determined by every set of columns of $M$ is equal to the row space of the principal submatrix lying in these columns.

Hermitian positive semidefinite matrices satisfy the PSRP. For the convenience of the reader, we reproduce the argument provided in [7] to prove this claim. Let $M \in \mathcal{P}_{N}(\mathbb{C})$ and write $M$ as

$$
M=\left(\begin{array}{cc}
A & B \\
B^{*} & C
\end{array}\right),
$$

where $A \in \mathcal{P}_{k}(\mathbb{C})$ and $1 \leq k<N$. It is enough to show that the row space of $\left(\begin{array}{c}A \\ B^{*}\end{array}\right)$ is equal to the row space of $A$. Working with orthogonal complements, this is equivalent to the following: If $\mathbf{v}=\left(\begin{array}{c}\mathbf{w} \\ \mathbf{0}\end{array}\right) \in \mathbb{C}^{N}$ and $A \mathbf{w}=\mathbf{0}$, then $M \mathbf{v}=\mathbf{0}$. But for such a vector $\mathbf{v}$ we have that $\mathbf{v}^{*} M \mathbf{v}=\mathbf{w}^{*} A \mathbf{w}=0$, and the result follows by the positive semidefiniteness of $M$.

We include below a short sketch of the proof from [7] to illustrate how the principal submatrix rank property is used in the proof of Theorem 2.4. The reader is referred to [7] for the details. 
Sketch of the proof of Theorem 2.4. The proof proceeds by induction, with the case $n=1$ trivial. Since the leading $(n-1) \times(n-1)$ principal submatrix $B$ of $A$ is positive semidefinite, there is a unitary monomial matrix $P$ such that $P^{-1} B P$ is a direct sum of $\mathbf{1}_{k}$ matrices and a zero matrix. If $R=P \oplus 1$, then $C=R^{-1} A R$ is such that all non-zero elements in the last row and column of $C$ are of modulus 1 and the leading $(n-1) \times(n-1)$ submatrix of $C$ is a direct sum of the desired form. We now partition the last row and column of $C$ in conformity with this direct sum. Since $C$ is positive semidefinite, it follows by the PSRP that each subvector of the last row and column of $C$ determined by this partition is a multiple of the vector with entries all 1 of the appropriate size by a number of modulus 1 or by 0 . The conclusion follows.

Next we show how Theorem 2.4 can naturally be extended to 3-PMP matrices.

Theorem 2.7. Given a Hermitian matrix $A \in \mathbb{C}^{N \times N}$, the following are equivalent.

(1) The matrix $A$ is 3-PMP with entries of modulus 0 or 1.

(2) There exist a diagonal matrix $D$, whose diagonal entries lie in $S^{1}$, and a permutation matrix $Q$, such that $(Q D)^{-1} A(Q D)$ is a block-diagonal matrix with each diagonal block a square matrix of either all ones or all zeros.

(3) The matrix $A \in \mathcal{P}_{N}(\mathbb{C})$ with entries of modulus 0 or 1.

Note that Theorem 2.7 fails for the Hermitian matrix

$$
A=\left(\begin{array}{rrr}
1 & 1 & -1 \\
1 & 1 & 1 \\
-1 & 1 & 1
\end{array}\right)
$$

which is 2-PMP but not 3-PMP. Thus Theorem 2.7 has no immediate generalization.

Proof of Theorem 2.7. If (2) holds then $(Q D)^{-1} A(Q D)$ is positive semidefinite and so $A$ is also positive semidefinite. Thus (2) implies (3). Clearly (3) implies (1).

Finally, suppose (1) holds. Then $a_{j j} \in\{0,1\}$ for all $j$, and the cases $N=1$ and $N=2$ are readily verified; suppose $N \geq 3$. Note that $A$ has no principal $3 \times 3$ submatrix of the form

$$
C=\left(\begin{array}{ccc}
1 & a & 0 \\
\bar{a} & 1 & b \\
0 & \bar{b} & 1
\end{array}\right), \quad \text { where } a, b \in S^{1},
$$

since $\operatorname{det} C=1-b \bar{b}-a \bar{a}=-1$. Thus the non-zero entries of $A$ can be permuted into a block-diagonal matrix by conjugation with a permutation matrix $Q$. It remains to show each non-zero diagonal block has rank one, since if these blocks are of the form $\mathbf{u}_{j} \mathbf{u}_{j}^{*}$, with the entries of $\mathbf{u}_{j}$ in $S^{1}$, then we can write the concatenation of the $\mathbf{u}_{j}$ as the diagonal entries of a diagonal matrix $D$, and the result follows.

We suppose henceforth that every entry of $A$ has modulus one, and claim that $A=\mathbf{u u}^{*}$, where $\mathbf{u}=\left(a_{11}, \ldots, a_{1 N}\right)^{*}$, i.e., that $a_{i j}=\overline{a_{1 i}} a_{1 j}$ for all $i$ and $j$. As $A$ is 3-PMP, the principal minor

$$
\left|\begin{array}{ccc}
1 & a_{1 i} & a_{1 j} \\
a_{i 1} & 1 & a_{i j} \\
a_{j 1} & a_{j i} & 1
\end{array}\right|=2 \Re\left(a_{1 i} a_{i j} a_{j 1}\right)-2
$$

is non-negative, so $a_{1 i} a_{i j} a_{j 1}=1$ and $a_{i j}=\overline{a_{1 i} a_{j 1}}=\overline{a_{1 i}} a_{1 j}$. This proves $(1) \Longrightarrow$ (2), and concludes the proof. 
Theorem 2.7 uses the 3-PMP property instead of the PSRP. It is therefore natural to ask how different these two properties are. To explore this question, we introduce a refinement of the principal submatrix rank property.

Definition 2.8. A matrix $M \in \mathbb{C}^{N \times N}$ is said to satisfy the $k$-principal submatrix rank property $(k-P S R P)$ if the following conditions hold.

(1) The column space determined by every set of $k$ rows of $M$ is equal to the column space of the principal submatrix lying in these rows.

(2) The row space determined by every set of $k$ columns of $M$ is equal to the row space of the principal submatrix lying in these columns.

Our next result shows that a $k$-PMP matrix satisfies the $(k-1)$-PSRP.

Theorem 2.9. Let $M \in \mathbb{C}^{N \times N}$ be a $k$-PMP Hermitian matrix, where $2 \leq k \leq N$. Then $M$ satisfies the $l$-PSRP for all $l<k$.

Proof. Since a $k$-PMP matrix is also $l$-PMP for all $l<k$, it suffices to take $l=k-1$. Without loss of generality, we show that the column space determined by the first $k-1$ rows of $M$ is equal to the column space of the leading principal $(k-1) \times(k-1)$ submatrix of $M$; the general case follows by simultaneously permuting the rows and columns of $M$. Write

$$
M=\left(\begin{array}{cc}
A & B \\
B^{*} & C
\end{array}\right)
$$

with $A \in \mathbb{C}^{(k-1) \times(k-1)}, B \in \mathbb{C}^{(k-1) \times(N-k+1)}$, and $C \in \mathbb{C}^{(N-k+1) \times(N-k+1)}$. Denote the columns of $B$ by $\mathbf{b}_{k}, \mathbf{b}_{k+1}, \ldots, \mathbf{b}_{N} \in \mathbb{C}^{k-1}$, and let $\operatorname{col}(A)$ denote the column space of $A$. We will prove that $\mathbf{b}_{i} \in \operatorname{col}(A)$ whenever $k \leq i \leq N$. Indeed, for such $i$, let

$$
M_{i}:=\left(\begin{array}{cc}
A & \mathbf{b}_{i} \\
\mathbf{b}_{i}^{*} & m_{i i}
\end{array}\right) \in \mathbb{C}^{k \times k}
$$

be the principal submatrix of $M$ formed by its rows and columns numbered $1, \ldots, k-1$, and $i$. By assumption, $M_{i} \in \mathcal{P}_{k}(\mathbb{C})$. If $m_{i i}=0$, then $\mathbf{b}_{i}=\mathbf{0} \in \operatorname{col}(A)$, which may be seen by inspecting $2 \times 2$ principal minors of $M_{i}$. Otherwise, the Schur complement of $m_{i i}$ in $M_{i}$ is positive semidefinite, i.e.,

$$
A-\frac{1}{m_{i i}} \mathbf{b}_{i} \mathbf{b}_{i}^{*} \in \mathcal{P}_{k-1}(\mathbb{C}) .
$$

If $\mathbf{b}_{i} \notin \operatorname{col}(A)$, there exists $\mathbf{v} \in \operatorname{col}(A)^{\perp}=\operatorname{ker} A$ such that $\mathbf{v}^{*} \mathbf{b}_{i} \neq 0$. For such a vector $\mathbf{v}$, we have that

$$
\mathbf{v}^{*}\left(A-\frac{1}{m_{i i}} \mathbf{b}_{i} \mathbf{b}_{i}^{*}\right) \mathbf{v}=-\frac{1}{m_{i i}}\left|\mathbf{v}^{*} \mathbf{b}_{i}\right|^{2}<0,
$$

contradicting Equation (2.1). We conclude that $\mathbf{b}_{i} \in \operatorname{col}(B)$, as claimed.

Theorem 2.9 shows that the $k$-PMP property imposes constraints on the row and column spaces of the matrix. However, our next result shows that there exist $k$-PMP matrices with an arbitrarily large gap between the dimension of the column space determined by a set of $l$ rows, and the rank of the principal submatrix lying in these rows. Thus there is a major discrepancy between the 3-PMP property and the PSRP.

Theorem 2.10. Let $2 \leq k \leq l<N$. Then there exists a real symmetric matrix

$$
M=\left(\begin{array}{cc}
A & B \\
B^{T} & C
\end{array}\right) \in \mathbb{R}^{N \times N},
$$

with $A \in \mathbb{R}^{l \times l}$, such that 
(1) the matrix $M$ is $k-P M P$,

(2) the matrix $A$ has rank $k-1$, and

(3) the matrix $\left(\begin{array}{ll}A & B\end{array}\right)$ has rank $\min \{l, k-1+N-l\}$.

In particular, the matrix $M$ does not satisfy the l-PSRP.

The following simple lemma is crucial to our proof of Theorem 2.10.

Lemma 2.11. Let $1 \leq m \leq l$. There exists a positive semidefinite matrix $A \in \mathcal{P}_{l}(\mathbb{R})$ with rank $m$, such that the $p \times p$ principal minors of $A$ are strictly positive whenever $1 \leq p \leq m$.

Proof. Let $\mathbf{u}=\left(u_{1}, \ldots, u_{l}\right)^{T} \in \mathbb{R}^{l}$ be a vector with distinct non-zero entries. Define

$$
A=\sum_{i=0}^{m-1} \mathbf{u}^{\circ i}\left(\mathbf{u}^{\circ i}\right)^{T},
$$

where $\mathbf{u}^{\circ i}$ is the vector with components $\left(u_{j}^{i}\right)_{1 \leq j \leq l}$. Then $A$ is positive semidefinite, and the desired properties follow immediately from the non-singularity of Vandermonde matrices.

Proof of Theorem 2.10. Let $A \in \mathcal{P}_{l}(\mathbb{R})$ be the matrix with rank $m=k-1$ provided by Lemma 2.11. Choose vectors $\mathbf{u}_{l+1}, \ldots, \mathbf{u}_{N} \in \operatorname{ker} A$ such that their span has the largest dimension possible, i.e., $\min \{l-k+1, N-l\}$. For $\epsilon_{l+1}, \ldots, \epsilon_{N}>0$, let $B$ be the matrix with columns $\epsilon_{l+1} \mathbf{u}_{l+1}, \ldots, \epsilon_{N} \mathbf{u}_{N}$. Also, let $C=\operatorname{Id}_{N-l}$, the $N$-l-dimensional identity matrix. Then the matrix $M$ given by Equation (2.2) satisfies properties (2) and (3) of the theorem.

We claim that $M$ is also $k$-PMP if $\epsilon_{l+1}, \ldots, \epsilon_{N}$ are small enough. To prove this claim, let $I:=\left\{i_{1}, \ldots, i_{k}\right\}$ be a subset of $\{1, \ldots, N\}$ of cardinality $k$, and let $M_{I}$ denote the principal submatrix of $M$ formed by restricting $M$ to the rows and columns in $I$. Clearly, $M_{I}$ is positive semidefinite if either $I \subset\{1, \ldots, l\}$ or $I \subset\{l+1, \ldots, N\}$. Now assume $I$ contains elements of both sets, so that $I=I_{1} \sqcup I_{2}$, with $I_{1} \subset\{1, \ldots, l\}, I_{2} \subset\{l+1, \ldots, N\}$, and both $I_{1}$ and $I_{2}$ are non-empty. Note that $M_{I_{2}}=\mathrm{Id}_{\left|I_{2}\right|}$. Thus $M_{I}$ is positive semidefinite as long as the Schur complement $M_{I_{1}}-M_{I_{1}, I_{2}} M_{I_{1}, I_{2}}^{T}$ is positive semidefinite, where $M_{I_{1}, I_{2}}$ denotes the submatrix of $M$ with rows in $I_{1}$ and columns in $I_{2}$. Note that $M_{I_{1}}=A_{I_{1}}$ is positive definite by Lemma 2.11. By a continuity argument, it follows that $M_{I_{1}}-M_{I_{1}, I_{2}} M_{I_{1}, I_{2}}^{T}$ is also positive definite if $0<\epsilon_{l+1}, \ldots, \epsilon_{N}<C_{I_{1}}$, for some positive constant $C_{I_{1}}$ that depends only on $I_{1}$. Let $C:=\min _{J:|J|<k} C_{J}$, where the minimum is taken over all subsets $J \subset\{1, \ldots, l\}$ of size less than $k$. Then $C>0$ since there are finitely many such subsets. If $0<\epsilon_{l+1}, \ldots, \epsilon_{N}<C$, this argument shows that $M_{I}$ is positive semidefinite for any set $I \subset\{1, \ldots, N\}$ of cardinality $k$. We conclude that $M$ is $k$-PMP, as required.

\section{PRincipal Minor POSITIVITy AND Signature}

We next explore how the property of a matrix being $k$-PMP affects its signature, and so its rank. In the most restrictive case, if an $N \times N$ matrix is $N$-PMP, then it has no negative eigenvalues; moreover, every admissible signature $\left(n_{+}, n_{0}=N-n_{+}, n_{-}=0\right)$ is attained, which may be seen by considering the block-diagonal matrix $\operatorname{Id}_{n_{+}} \oplus \mathbf{0}_{n_{0}}$.

The purpose of this section is to consider all other Hermitian matrices. We now prove:

Theorem 3.1. Fix integers $k$ and $N$, with $0 \leq k<N$.

(1) Let $A \in \mathbb{C}^{N \times N}$ be $k$-PMP but not $(k+1)$-PMP. Then $A$ has at least $k$ positive eigenvalues, and at least one negative eigenvalue. 
(2) Conversely, for every signature $\left(n_{+}, n_{0}, n_{-}\right)$such that

$$
n_{+} \geq k, \quad n_{0}=N-n_{+}-n_{-} \geq 0, \quad \text { and } \quad n_{-} \geq 1,
$$

there exists a matrix $A \in \mathbb{C}^{N \times N}$ with this signature, and such that $A$ is $k$-PMP but not $(k+1)-P M P$.

Proof. We begin by showing (1). By assumption, there exists a $(k+1) \times(k+1)$ principal minor of $A$, say $B$, such that $\operatorname{det} B<0$. As $B$ is $k$-PMP, it follows by Cauchy interlacing that $B$ has one negative eigenvalue and $k$ positive ones. Another application of Cauchy interlacing now gives the same statement for $A$.

We next prove the converse result (2). By Lemma 2.11, there exists a matrix $B \in \mathcal{P}_{k+n_{-}}(\mathbb{R})$ of rank $k$ such that all $p \times p$ principal minors of $B$ are strictly positive, for $1 \leq p \leq k$. Now let $P \in \mathcal{P}_{k+n_{-}}(\mathbb{R})$ denote the projection matrix of the subspace ker $B$, and define

$$
B_{\epsilon}:=B-\epsilon P, \quad \text { where } \epsilon>0 .
$$

By the continuity of determinants, we may fix $\epsilon$ sufficiently small to ensure that all $p \times p$ principal minors of $B_{\epsilon}$ are positive, for $1 \leq p \leq k$, so that $B_{\epsilon}$ is $k$-PMP. Furthermore, it is clear by diagonalization that $B_{\epsilon}$ has signature $\left(k, 0, n_{-}\right)$.

We now show that $B_{\epsilon}$ is not $(k+1)$-PMP. In fact, we claim that every $(k+1) \times(k+1)$ principal minor of $B_{\epsilon}$ is negative. If $\operatorname{det} M \geq 0$ for some $(k+1) \times(k+1)$ principal minor $M$ of $B_{\epsilon}$, then $M$ is positive semidefinite and so has $k+1$ non-negative eigenvalues. This last statement then holds for $B_{\epsilon}$, by Cauchy interlacing, which contradicts the fact that $B_{\epsilon}$ has signature $\left(k, 0, n_{-}\right)$.

The result now follows by taking $A:=B_{\epsilon} \oplus \operatorname{Id}_{n_{+}-k} \oplus \mathbf{0}_{N-n_{+}-n_{-}}$.

\section{Schubert Cell-type Stratification of 3-PMP Matrices}

In this section we explain and generalize the novel Schubert cell-type stratification of $\mathcal{P}_{N}(\mathbb{C})$ uncovered in [2], which plays a crucial role in determining the simultaneous kernel $\mathcal{K}(A)$ defined in Equation (1.1).

Theorem 4.1 ([2, Theorem 5.1]). Fix a multiplicative subgroup $G \subset \mathbb{C}^{\times}$, an integer $N \geq 1$, and a non-zero matrix $A \in \mathcal{P}_{N}(\mathbb{C})$.

(1) Suppose $\left\{I_{1}, \ldots, I_{m}\right\}$ is a partition of $\{1, \ldots, N\}$ satisfying the following conditions.

(a) Each diagonal block $A_{I_{j}}$ of $A$ has rank at most one, and $A_{I_{j}}=\mathbf{u}_{j} \mathbf{u}_{j}^{*}$ for a unique vector $\mathbf{u}_{j} \in \mathbb{C}^{\left|I_{j}\right|}$ with first entry $\mathbf{u}_{j, 1} \in[0, \infty)$.

(b) The entries of each diagonal block $A_{I_{j}}$ lie in a single $G$-orbit.

Then there exists a unique matrix $C=\left(c_{i j}\right)_{i, j=1}^{m}$ such that $c_{i j}=0$ unless $\mathbf{u}_{i} \neq 0$ and $\mathbf{u}_{j} \neq 0$, and $A$ is a block matrix with

$$
A_{I_{i} \times I_{j}}=c_{i j} \mathbf{u}_{i} \mathbf{u}_{j}^{*} \quad(1 \leq i, j \leq m) .
$$

Moreover, the entries of each off-diagonal block of $A$ also lie in a single $G$-orbit. The matrix $C \in \mathcal{P}_{m}(\bar{D}(0,1))$, and the matrices $A$ and $C$ have equal rank.

(2) Consider the condition (c).

(c) The diagonal blocks of A have maximal size, i.e., each diagonal block is not contained in a larger diagonal block that has rank one.

There exists a partition $\left\{I_{1}, \ldots, I_{m}\right\}$ such that (a), (b) and (c) hold, and such a partition is unique up to relabelling of the indices.

(3) Suppose (a), (b), and (c) hold, and $G=\mathbb{C}^{\times}$. Then the off-diagonal entries of $C$ lie in the open disc $D(0,1)$. 
(4) If $G \subset S^{1}$, then diagonal blocks of $A$ in a single $G$-orbit have rank at most one.

Note that Theorem 4.1(4) is an immediate consequence of Theorem 2.4.

Example 4.2. To illustrate Theorem 4.1, consider the following $5 \times 5$ Hermitian matrix,

$$
A=\left(\begin{array}{ccccc}
2 & 2 & 1 & -2 i & 2 \\
2 & 2 & 1 & -2 i & 2 \\
1 & 1 & 1 & -i & 1 \\
2 i & 2 i & i & 2 & 2 i \\
2 & 2 & 1 & -2 i & 2
\end{array}\right)
$$

It is readily verified that $A \in \mathcal{P}_{5}(\mathbb{C})$. Let $G_{1}=\{1\}$, the trivial multiplicative subgroup of $\mathbb{C}^{\times}$, and consider the partition $\pi_{1}:=\{\{1,2,5\},\{3\},\{4\}\}$. Permuting the rows and columns of $A$ according to $\pi_{1}$, we obtain

$$
A^{\prime}:=\left(\begin{array}{ccccc}
2 & 2 & 2 & 1 & -2 i \\
2 & 2 & 2 & 1 & -2 i \\
2 & 2 & 2 & 1 & -2 i \\
1 & 1 & 1 & 1 & -i \\
2 i & 2 i & 2 i & i & 2
\end{array}\right)
$$

It follows immediately that $\pi_{1}$ is the unique partition, up to relabelling of the indices, afforded by Theorem 4.1. Similarly, consider the cyclic subgroup $G_{2}=\{1,-1, i,-i\} \subset \mathbb{C}^{\times}$, and let the partition $\pi_{2}:=\{\{1,2,4,5\},\{3\}\}$. Permuting the rows and columns of $A$ with respect to $\pi_{2}$, we obtain

$$
A^{\prime \prime}:=\left(\begin{array}{ccccc}
2 & 2 & -2 i & 2 & 1 \\
2 & 2 & -2 i & 2 & 1 \\
2 i & 2 i & 2 & 2 i & i \\
2 & 2 & -2 i & 2 & 1 \\
1 & 1 & -i & 1 & 1
\end{array}\right) .
$$

The $4 \times 4$ leading principal submatrix of $A^{\prime \prime}$ has rank 1 , and its entries lie in the same $G_{2^{-}}$ orbit. It follows that $\pi_{2}$ is the partition provided by Theorem 4.1 in this case. Finally, if we take $G_{3}=S^{1} \subset \mathbb{C}^{\times}$, then we recover the same block structure and partition as for $G_{2}$.

For all of these subgroups, the entries of each off-diagonal block belong to a single orbit, as guaranteed by the theorem.

Theorem 4.1 provides a natural stratification of the cone $\mathcal{P}_{N}(\mathbb{C})$.

Definition 4.3. Let $\Pi_{N}$ denote the set of all partitions of $\{1, \ldots, N\}$, partially ordered so that $\pi^{\prime} \prec \pi$ if and only if $\pi$ is a refinement of $\pi^{\prime}$. Given a matrix $A \in \mathcal{P}_{N}(\mathbb{C})$ and $G \subset S^{1}$, define $\pi^{G}(A) \in \Pi_{N}$ to be the partition provided by Theorem 4.1 for the matrix $A$. Conversely, for a partition $\pi \in \Pi_{N}$ and $G \subset S^{1}$, let the stratum

$$
\mathcal{S}_{\pi}^{G}:=\left\{A \in \mathcal{P}_{N}(\mathbb{C}): \pi^{G}(A)=\pi\right\} .
$$

The Schubert property is reflected in the decomposition $\overline{\mathcal{S}_{\pi}^{G}}=\bigsqcup_{\pi^{\prime} \prec \pi} \mathcal{S}_{\pi^{\prime}}^{G}$, where $\pi^{\prime}$ runs over all coarsenings of $\pi$ in $\Pi_{N}$.

In [2, Section 5], it was shown that for any matrix $A \in \mathcal{P}_{N}(\mathbb{C})$, the simultaneous kernel of its entrywise powers,

$$
\mathcal{K}(A):=\bigcap_{n \geq 0} \operatorname{ker} A^{\circ n}
$$


is explicitly computable, and equals the kernel of a single matrix that depends on $A$ only through the partition $\pi^{\{1\}}(A)$; see Theorem 1.1. Consequently, this simultaneous kernel $\mathcal{K}(A)$ is unchanged as $A$ runs through a fixed stratum $\mathcal{S}_{\pi}^{\{1\}}$ in $\mathcal{P}_{N}(\mathbb{C})$.

In order to prove a version of Theorem 1.1 for 3-PMP matrices, we first extend Theorem 4.1,

Theorem 4.4. Suppose $G \subset \mathbb{C}^{\times}$is a multiplicative subgroup and $3 \leq k \leq N$. The assertions in Theorem 4.1 all hold if $A$ is only required to be $k$-PMP, except that the matrix $C$ is now only assured to be $k$-PMP, rather than positive semidefinite.

Proof. All assertions but the last follow as in the proof of Theorem 4.1 given in 2], using the 3-PMP property and Theorem 2.7 in place of Theorem 2.4 for (4). That $C$ inherits the $k$-PMP property from $A$ is readily verified.

We will refer to the analogous statement to Theorem 4.1(1) provided by Theorem 4.4 as Theorem 4.4(1), and similarly for the other parts of these theorems.

Definition 4.5. Suppose $G \subset \mathbb{C}^{\times}$is a multiplicative subgroup and $A \in \mathbb{C}^{N \times N}$ is $k$-PMP, where $3 \leq k \leq N$. The partition given by Theorem 4.4 (2) is denoted $\pi^{G}(A)$.

Theorem 4.4 immediately leads to stratifications of the $k$-PMP matrices, for $3 \leq k \leq N$. These stratifications respect the natural inclusions, so that $\pi^{G}(A)$ is independent of $k$.

This common Schubert cell-type stratification in fact holds more generally, for all Hermitian matrices.

Proposition 4.6. Fix a multiplicative subgroup $G \subset \mathbb{C}^{\times}$, an integer $N \geq 1$, and a Hermitian matrix $A \in \mathbb{C}^{N \times N}$. There exists a coarsest partition $\pi_{\min }(A)=\left\{I_{1}, \ldots, I_{m}\right\} \in \Pi_{N}$, such that the entries of the block submatrix $A_{I_{i} \times I_{j}}$ lie in a single $G$-orbit, for all $i, j \in\{1, \ldots, m\}$. This partition is unique up to relabelling of the indices.

If, moreover, $A$ is 3-PMP and $G \subset S^{1}$, then $\pi_{\min }(A)=\pi^{G}(A)$.

Note that 3-PMP matrices differ from Hermitian matrices which are not 3-PMP, in that even for $G \subset S^{1}$, every block given by the partition $\pi_{\min }$ can have rank greater than 1 . For example, the following Hermitian matrix $A$ is 2-PMP but not 3-PMP, and for $G=\{1,-1\}$ we have that $\pi_{\min }(A)=\{\{1,2,3\},\{4,5,6\}\}$, with all four blocks of $A$ non-singular:

$$
A=\left(\begin{array}{rrrrrr}
2 & 2 & -2 & 1 & 1 & -1 \\
2 & 2 & 2 & 1 & 1 & 1 \\
-2 & 2 & 2 & -1 & 1 & 1 \\
1 & 1 & -1 & 2 & 2 & -2 \\
1 & 1 & 1 & 2 & 2 & 2 \\
-1 & 1 & 1 & -2 & 2 & 2
\end{array}\right) .
$$

Proof of Proposition 4.6. The non-trivial part is to establish uniqueness. To do so, we first claim that if $\pi_{1}, \pi_{2} \in \Pi_{N}$ satisfy the property in the assertion, then so does their meet $\pi_{1} \wedge \pi_{2}$; note this gives uniqueness, since minimal $\pi_{1}$ and $\pi_{2}$ are such that $\pi_{1}=\pi_{1} \wedge \pi_{2}=\pi_{2}$.

To show the claim, first recall that the meet $\pi_{1} \wedge \pi_{2}$ can be constructed as follows: connect vertices $i, i^{\prime} \in\{1, \ldots, N\}$ by an edge if they lie in the same block of $\pi_{1}$ or $\pi_{2}$; this defines a graph whose connected components are the blocks of the partition $\pi_{1} \wedge \pi_{2}$. Denote this equivalence relation by $i \sim i^{\prime}$ in $\pi_{1} \wedge \pi_{2}$.

Now suppose $i \sim i^{\prime}$ and $j \sim j^{\prime}$ in $\pi_{1} \wedge \pi_{2}$, so there are paths joining them, each of whose vertices lies in a block of $\pi_{1}$ or $\pi_{2}$. Denote these paths by

$$
i=i_{0} \leftrightarrow i_{1} \leftrightarrow \cdots \leftrightarrow i_{r}=i^{\prime} \quad \text { and } \quad j=j_{0} \leftrightarrow j_{1} \leftrightarrow \cdots \leftrightarrow j_{s}=j^{\prime} .
$$


We claim that $a_{i j} \in G \cdot a_{i^{\prime} j^{\prime}}$. Indeed, using the above paths,

$$
a_{i j}=a_{i_{0} j} \in G a_{i_{1} j}=G a_{i_{2} j}=\cdots=G a_{i_{r} j}=G a_{i^{\prime} j_{0}}=G a_{i^{\prime} j_{1}}=\cdots=G a_{i^{\prime} j^{\prime}} .
$$

This proves the claim, and hence all but the last assertion.

If $A$ is 3-PMP and $G \subset S^{1}$, then Theorem 2.7 gives that $\pi_{\min }(A)$ satisfies properties (a) and (b) of Theorem 4.4. Hence $\pi_{\min }(A)$ is finer than $\pi^{G}(A)$, by property (c). Conversely, by Theorem 4.4(1), each block of $A$ given by $\pi^{G}(A)$ lies in a single $G$-orbit, so $\pi^{G}(A)$ is finer than $\pi_{\min }(A)$. Thus $\pi_{\min }(A)=\pi^{G}(A)$, as required.

The preceding result allows us to extend the Schubert cell-type stratification to all $N \times N$ Hermitian matrices.

Definition 4.7. Given $N \geq 1, G \subset \mathbb{C}^{\times}$, and a Hermitian matrix $A \in \mathbb{C}^{N \times N}$, define the partition $\pi^{G}(A) \in \Pi_{N}$ to be $\pi_{\min }(A)$, as in Proposition 4.6. Furthermore, given $\pi \in \Pi_{N}$, let

$$
\mathcal{S}_{\pi}^{G}:=\left\{\text { Hermitian } A \in \mathbb{C}^{N \times N}: \pi^{G}(A)=\pi\right\} .
$$

Once again, we have that $\overline{\mathcal{S}_{\pi}^{G}}=\bigsqcup_{\pi^{\prime} \prec \pi} \mathcal{S}_{\pi^{\prime}}^{G}$ for all $\pi \in \Pi_{N}$ and $G \subset \mathbb{C}^{\times}$, as above.

Remark 4.8. Let $N \geq 3$. The matrix

$$
A=\left(\begin{array}{ccc}
1 & 2 & 0 \\
2 & 8 & 0 \\
0 & 0 & \operatorname{Id}_{N-2}
\end{array}\right)
$$

is positive semidefinite, so 3-PMP. If $G=\langle 2\rangle=\left\{2^{n}: n \in \mathbb{Z}\right\}$, then $\pi_{\min }(A)=\{\{1,2\},\{3\}\}$ and the diagonal block $A_{\{1,2\}}$ has rank 2. Thus $\pi_{\min }(A) \neq \pi^{G}(A)$ and the final part of Proposition 4.6 has no immediate extension beyond the case where $G \subset S^{1}$.

\section{The simultaneous Kernels of HADAMARD POWERS OF 3-PMP MATRICES}

Having understood the Schubert cell-type stratification of the class of 3-PMP matrices, our aim below is to compute the simultaneous kernel of the entrywise powers of any 3-PMP matrix. This extends [2, Theorem 5.7], which was obtained for positive semidefinite matrices only.

Theorem 5.1. Let the Hermitian matrix $A \in \mathbb{C}^{N \times N}$ be $3-P M P$, and let $\pi^{\prime}=\left\{I_{1}^{\prime}, \ldots, I_{m^{\prime}}^{\prime}\right\}$ be any partition refined by $\pi=\pi^{\{1\}}(A)=\left\{I_{1}, \ldots, I_{m}\right\}$. The following spaces are equal.

(1) The simultaneous kernel of $\mathbf{1}_{N}, A, \ldots, A^{\circ(N-1)}$.

(2) The simultaneous kernel of $A^{\text {on }}$ for all $n \geq 0$.

(3) The simultaneous kernel of the block-diagonal matrices $\operatorname{diag} A_{\pi^{\prime}}^{\circ n}:=\oplus_{j=1}^{m^{\prime}} A_{I_{j}^{\prime} \times I_{j}^{\prime}}^{\circ n}$ for all $n \geq 0$.

(4) The kernel of $J_{\pi}:=\oplus_{j=1}^{m} \mathbf{1}_{I_{j}}$.

However, this equality of kernels need not hold for matrices that are not 3-PMP.

Proof. That (1) and (2) describe the same subspace follows from [2, Lemma 3.5], which gives that $A^{\circ M}=\sum_{j=0}^{N-1} D_{M, j}(A) A^{\circ j}$ for certain matrices $D_{M, j}(A)$ and any $M \geq 0$. We now show equality of the subspaces (2) and (4); the same argument, mutatis mutandis, implies that (2) and (3) are equal as well.

Note first that $\mathbf{v}=\left(v_{1}, \ldots, v_{N}\right)^{T} \in$ ker $J_{\pi}$ if and only if $\sum_{i \in I_{j}} v_{i}=0$ for all $j \in\{1, \ldots, m\}$. Since $A^{\text {on }}$ is constant on each block of the form $I_{i} \times I_{j}$, it follows that $\operatorname{ker} J_{\pi} \subseteq \operatorname{ker} A^{\text {on }}$ for all $n \geq 0$. 
To obtain the reverse inclusion, let $B \in \mathbb{C}^{m \times m}$ denote the compression of $A$,

$$
B:=\Sigma_{\pi}^{\downarrow}(A),
$$

so that $b_{i j}$ equals the constant value taken in the block $A_{I_{i} \times I_{j}}$. Let $\mathbf{v} \in \cap_{n \geq 0} \operatorname{ker} A^{\text {on }}$ and write $\mathbf{w}:=\left(w_{1}, \ldots, w_{m}\right)^{T}$, with $w_{j}:=\sum_{i \in I_{j}} v_{i}$ for all $j$. Then $\mathbf{v} \in$ ker $J_{\pi}$ if and only if $\mathbf{w}=\mathbf{0}$. Since $A^{\circ n} \mathbf{v}=\mathbf{0}$ for all $n$, it follows that $B^{\circ n} \mathbf{w}=\mathbf{0}$ for all $n \geq 0$. Thus the result follows if $\cap_{n \geq 0}$ ker $B^{\circ n}=\{0\}$.

If $(p, q) \in I_{i} \times I_{j}$ then $b_{i j}=a_{p q}$; in particular, $b_{i i} \geq 0$, and we may assume without loss of generality that the diagonal entries of $B$ are in non-increasing order. Supposing for contradiction that $b_{i i}=b_{i j}$, where $j>i$, the 3-PMP property for $A$ gives that

$$
b_{i i}^{2} \geq b_{i i} b_{j j}=a_{p p} a_{q q} \geq\left|a_{p q}\right|^{2}=\left|b_{i j}\right|^{2}=b_{i j}^{2}=b_{i i}^{2},
$$

so $b_{i i}=b_{i j}=b_{j i}=b_{j j}$. This shows that $B$ is constant on the block $\{i, j\} \times\{i, j\}$, which contradicts the choice of $\pi$, by Theorem 4.4. Hence we have that

$$
b_{i i} \neq b_{i j} \quad \text { whenever } 1 \leq i<j \leq m .
$$

We claim this implies that

$$
\bigcap_{n=0}^{m-1} \operatorname{ker} B^{\circ n}=\{\mathbf{0}\},
$$

which will establish the desired conclusion.

We proceed by induction on $m$, with the base case $m=1$ being clear, since $B^{\circ 0}=(1)$. Suppose $\mathbf{u}=\left(u_{j}\right) \in \mathbb{C}^{m}$ is such that $B^{\circ n} \mathbf{u}=\mathbf{0}$ whenever $0 \leq n \leq m-1$. If we can show that $u_{1}=0$, then the result follows by a standard induction argument.

It suffices to assume that $\mathbf{u}$ is annihilated by $\mathbf{r}^{\circ 0}, \ldots, \mathbf{r}^{\circ(m-1)}$, where $\mathbf{r}:=\left(b_{11}, \ldots, b_{1 m}\right)$ is the first row of $B$. Let $\mathbf{s}:=\left(s_{1}, \ldots, s_{k}\right)$ be a compression of $\mathbf{r}$, which contains each distinct entry in $\mathbf{r}$ exactly once, and has $s_{1}=b_{11}$. Suppose $\{1, \ldots, m\}=I_{1}^{\prime \prime} \sqcup \cdots \sqcup I_{k}^{\prime \prime}$ is the corresponding partition, so that

$$
s_{j}=b_{1 i} \quad \Longleftrightarrow \quad i \in I_{j}^{\prime \prime} \quad(j=1, \ldots, k),
$$

and note that $I_{1}^{\prime \prime}=\{1\}$. If $v_{j}:=\sum_{i \in I_{j}^{\prime}} u_{i}$, then the vector $\mathbf{v}=\left(v_{1}, \ldots, v_{k}\right)^{T}$ is annihilated by $\mathbf{s}^{\circ 0}, \ldots, \mathbf{s}^{\circ(m-1)}$. The first $k$ of these are linearly independent, as they form a Vandermonde matrix, so $\mathbf{v}=\mathbf{0}$. Hence $u_{1}=v_{1}=0$, as required.

Finally, to see that the inclusion between the kernels in (4) and (2) is not always an equality, consider the Toeplitz tridiagonal matrix $T_{N}$, with $(i, j)$ entry equal to 1 if $|i-j| \leq 1$ and equal to 0 otherwise, for $N \geq 3$. This matrix is readily seen to be 2-PMP but not 3-PMP. Note that $\pi=\pi^{\{1\}}\left(T_{N}\right)=\{\{1\}, \ldots,\{N\}\}$, so ker $J_{\pi}=\{0\}$. However, if $N=3 k+2$ for some $k \geq 1$, then $(1,-1,0,1,-1,0, \ldots, 1,-1)^{T}$ lies in $\operatorname{ker} \mathbf{1}_{N}$ and $\operatorname{ker} T_{N}^{\circ n}$ for all $n \geq 1$.

Remark 5.2. A more general objective is the computation of the simultaneous kernel of the set of Hadamard powers $\left\{A^{\circ n}: n \geq 0\right\}$, where $A \in \mathbb{F}^{M \times N}$ is a linear map between finitedimensional vector spaces over a field $\mathbb{F}$. Clearly this reduces to finding the simultaneous kernels of the Hadamard powers of each row $\mathbf{u}^{T}$ of $A$. There exists a unique coarsest partition $\left\{I_{1}, \ldots, I_{m}\right\} \in \Pi_{N}$ such that the entries in $\mathbf{u}$ are equal within each block $I_{j}$, for all rows $\mathbf{u}^{T}$ of $A$. Working as in the proof of Theorem 5.1 shows that

$$
\bigcap_{\mathbf{u}^{T}} \bigcap_{n \geq 0} \operatorname{ker}\left(\mathbf{u}^{T}\right)^{\circ n}=\bigoplus_{j=1}^{m} \operatorname{ker} \mathbf{1}_{1 \times I_{j}} .
$$


This provides a recipe to compute the simultaneous kernel of the Hadamard powers of an arbitrary rectangular matrix over any field. The Toeplitz counterexample $T_{N}$ in the proof of Theorem 5.1 shows that, in order to give a more precise description of the simultaneous kernel, additional assumptions are required, such as being 3-PMP.

In light of this remark, it is noteworthy that the proof of Theorem 5.1 gives the following result.

Proposition 5.3. Let $\mathbb{F}$ be an arbitrary field, and suppose $A \in \mathbb{F}^{N \times N}$ is such that

$$
a_{i i} \neq a_{i j} \quad \text { whenever } 1 \leq i<j \leq N .
$$

Then $\cap_{n=0}^{N-1} \operatorname{ker} A^{\text {on }}=\{\mathbf{0}\}$.

Remark 5.4. The operator $\Sigma_{\pi}^{\downarrow}(\cdot)$ in Equation (5.1) has a number of interesting properties. These features and their ramifications will be explored in forthcoming work [4].

Acknowledgments. The authors thank the International Centre for Mathematical Sciences, Edinburgh, and Iowa State University, host organization of the 2017 ILAS conference, for their hospitality, stimulating atmosphere, and excellent working conditions. D.G. is partially supported by a University of Delaware Research Foundation grant, and by a Simons Foundation collaboration grant for mathematicians.

\section{REFERENCES}

[1] Stefan Banach. Théorie des opérations linéaires. (French) Monografje Matematyczne 1, Warszawa, 1932.

[2] Alexander Belton, Dominique Guillot, Apoorva Khare, and Mihai Putinar. Matrix positivity preservers in fixed dimension. I. Adv. Math. 298:325-368, 2016.

[3] Alexander Belton, Dominique Guillot, Apoorva Khare, and Mihai Putinar. Schur polynomials and matrix positivity preservers. FPSAC '16 Proceedings (DMTCS), 155-166, 2016.

[4] Alexander Belton, Dominique Guillot, Apoorva Khare, and Mihai Putinar. Matrix positivity preservers in fixed dimension. II. To appear.

[5] Andries E. Brouwer and Willem H. Haemers. Spectra of graphs. Springer, New York, 2012.

[6] Dominique Guillot and Bala Rajaratnam. Retaining positive definiteness in thresholded matrices. Linear Algebra Appl., 436(11):4143-4160, 2012.

[7] Daniel Hershkowitz, Michael Neumann, and Hans Schneider. Hermitian positive semidefinite matrices whose entries are 0 or 1 in modulus. Linear Multilinear Algebra, 46(4):259-264, 1999.

[8] Roger A. Horn. The theory of infinitely divisible matrices and kernels. Trans. Amer. Math. Soc., 136:269$286,1969$.

[9] John Lamperti. On the isometries of certain function-spaces. Pacific J. Math. 8(3):459-466, 1958.

[10] Srinivasa Raghavan Mohan, Thiruvenkatachari Parthasarathy, and Raghavendra Sridhar. The linear complementarity problem with exact order matrices. Math. Oper. Res. 19(3):618-644, 1994.

(A. Belton) Department of Mathematics and Statistics, Lancaster University, Lancaster, UK E-mail address: a.belton@lancaster.ac.uk

(D. Guillot) University of Delaware, Newark, DE, USA

E-mail address: dguillot@udel.edu

(A. Khare) Indian Institute of Science, Bangalore, India

E-mail address: khare@iisc.ac.in

(M. Putinar) University of California at Santa Barbara, CA, USA and Newcastle University, NewCastle upon Tyne, UK

E-mail address: mputinar@math.ucsb.edu, mihai.putinar@ncl.ac.uk 